\title{
A POSSIBLE MANIFESTATION OF MICROLENSING IN PULSAR TIMING
}

\author{
T.I.LARCHENKOVA AND O.V.DOROSHENKO \\ Astro Space Center of P.N.Lebedev Physical Institute \\ Profsoyuznaya 84/32, Moscow 117810 RUSSIA
}

\begin{abstract}
Gravitational lensing and the time delay of a pulsar signal in the gravitational field of a mass are General Relativistic effects that may be used as a tool to detect the observational parameters of dark matter in our Galaxy. We propose to use observations of the time delay of pulses from pulsars to detect lensing objects located close to the line of the sight, to study the distribution of dark matter in our Galaxy. We discuss the possibility of finding such an event by measuring the delay of pulses from a pulsar, and apply it to data for PSR B0525+21.
\end{abstract}

\section{Time delay and application to the pulsar B0525+21}

Several single pulsars exhibit some unexplained distortions of the observed times-of-arrival (TOA) of their pulses. The differences between the observed TOAs and those calculated using the classical spin-down model of the pulsar rotation are the residuals of the TOA. The residuals are believed to be caused by some instability in the pulsar rotation. They might also be caused by a mass moving close to the line of the sight. The propagation delay of radio signals in the gravitational field of a massive object (the Shapiro effect) causes a sharp growth in amplitude of the TOA near the conjunction of the mass with the line of sight of the observer.

We analyzed the timing data for several pulsars obtained at the JPL by Downs \& Reichley (1983) and Downs \& Krause-Polstroff (1986) from 1968 to 1983 . The data on the arrival times were reduced using the standard fit of the pulsar astrometric and spin parameters based upon the data reduction algorithm developed by Doroshenko \& Kopeikin (1990). From an analysis of the observed residuals we concluded that amongst the studied objects 


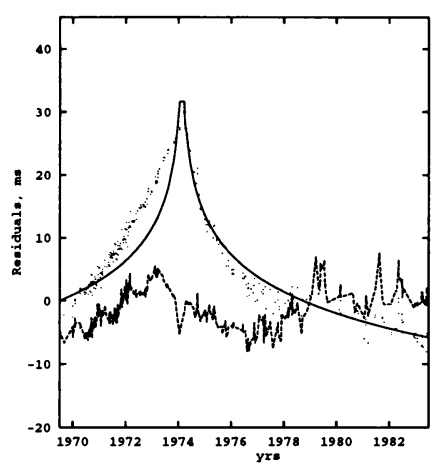

Figure 1. The TOA residuals for PSR B0525+21. The circles show the observed TOA residuals after fitting for the pulsar spin and astrometric parameters. The solid line is the best fit Shapiro delay for a lensing mass. The dashed line is the post-fit TOA residuals.

PSR B0525+21 may present a case of micro-lensing. After subtracting the best fitting polynomial to the arrival times, we observe significant TOA residuals with a behavior similar to that caused by a mass passing close to the line of sight. The observed residuals of the TOAs of the pulsar are shown as the small circles on the Fig.1.

Our fitted values for the mass and other parameters of the lensing object are found to be: $M=330 \pm 50 \mathrm{M}_{\odot} ; V_{P} / d=(1.0 \pm 0.7) \times 10^{-7} \mathrm{~s}^{-1}$; $T_{0}=2442040.0 \mathrm{JD}$, where $M$ is the lens mass, $V_{P} / d$ is the ratio of the relative velocity of the pulsar to the impact parameter, and $T_{0}$ is the time of conjunction. With these values of the parameters the pre-fit and post-fit residuals are equal to $15 \mathrm{~ms}$ and $3 \mathrm{~ms}$ respectively. The results are shown in Figure 1.

Because pulsars are the fastest objects in Galaxy, we can suppose that the pulsar velocity is larger than the lensing object velocity. For B $0525+21$ $V_{P} \sim \mu R \approx 200 \mathrm{~km} \mathrm{~s}^{-1}$, so that our value for $V_{P} / d$ gives the distance of closest approach $d \approx 13 \mathrm{AU}$. One can suggest that the observed extra modulation is due to a time delay PSR B0525+21 caused by a mass $M=$ $330 \mathrm{M}_{\odot}$ passing near the line of the sight. We suggest that the gravitating mass may be a robust association of massive baryonic objects (Moore et al. 1995) or a black hole.

\section{References}

Doroshenko, O.V. \& Kopeikin, S.M., 1990, SvA, 34(5), 496

Downs, G.S. \& Reichley, P.E., 1983, ApJS, 53, 169

Downs, G.S. \& Krause-Polstroff, J., 1986, ApJS, 62, 81

Moore, B. \& Silk, J., 1995, ApJL, 442, L5 